\title{
CONTINUOUS-STATE BRANCHING PROCESSES AND SELF-SIMILARITY
}

\author{
A. E. KYPRIANOU $* * *$ AND \\ J. C. PARDO, ${ }^{* * *}$ University of Bath
}

\begin{abstract}
In this paper we study the $\alpha$-stable continuous-state branching processes (for $\alpha \in(1,2]$ ) and the $\alpha$-stable continuous-state branching processes conditioned never to become extinct in the light of positive self-similarity. Understanding the interaction of the Lamperti transformation for continuous-state branching processes and the Lamperti transformation for positive, self-similar Markov processes gives access to a number of explicit results concerning the paths of $\alpha$-stable continuous-state branching processes and $\alpha$-stable continuous-state branching processes conditioned never to become extinct.
\end{abstract}

Keywords: Positive, self-similar Markov process; Lamperti representation; stable Lévy process; conditioning to stay positive; continuous-state branching process

2000 Mathematics Subject Classification: Primary 60G18; 60G51; 60B52

\section{Introduction}

This paper is principally concerned with positive, self-similar Markov processes (PSSMPs) which are also either continuous-state branching processes or continuous-state branching processes with immigration. All of the aforementioned processes shall be defined in more detail in the next section. However, for the purpose of giving a brief sense of the main goals of this paper in this section, we briefly recall the following.

A PSSMP $X=\left(X_{t}, t \geq 0\right)$ has the defining property that it is a nonnegative-valued strong Markov process with probabilities $\left(\mathrm{Q}_{x}, x \geq 0\right)$ such that, for each $k>0$,

$$
\text { the law of }\left(k X_{k^{-\alpha} t}, t \geq 0\right) \text { under } \mathrm{Q}_{x} \text { is given by } \mathrm{Q}_{k x} \text {, }
$$

where $\alpha>0$ is a constant known as the index of self-similarity. A continuous-state branching process (CSBP) on the other hand is a nonnegative-valued strong Markov process with probabilities $\left(\mathrm{P}_{x}, x \geq 0\right)$ such that, for any $x, y \geq 0, \mathrm{P}_{x+y}$ is equal in law to the convolution of $\mathrm{P}_{x}$ and $\mathrm{P}_{y}$. CSBPs may be thought of as the continuous (in time and space) analogues of classical Bienaymé-Galton-Watson branching processes. Associated with a CSBP $\left(Y, \mathrm{P}_{x}\right)$ is its Malthusian parameter, $-\rho$, which characterises the mean rate of growth in the sense that $\mathbb{E}_{x}\left(Y_{t}\right)=x \mathrm{e}^{-\rho t}$ for all $t \geq 0$. The PSSMPs, which are simultaneously CSBPs, that we shall consider are critical in the sense that $\rho=0$ and they are closely related, via a path transformation, to spectrally positive $\alpha$-stable Lévy processes for $\alpha \in(1,2]$. Being critical processes, standard theory allows us to talk about CSBPs conditioned never to become extinct in an appropriate sense. Roughly speaking, we may think of the latter as the result of a Doob

Received 11 December 2007; revision received 20 October 2008.

* Postal address: Department of Mathematical Sciences, University of Bath, Claverton Down, Bath BA2 7AY, UK.

** Email address: a.kyprianou@bath.ac.uk

*** Email address: jcpm20@bath.ac.uk 
$h$-transform of the law of a CSBP with $h(x)=x$. Owing to the recent works of Lambert [15], [17], it is known that in the case in which $\rho=0$, a CSBP conditioned never to become extinct also corresponds, in an appropriate sense, to a CSBP with immigration. We shall see that, when the underlying CSBP is a PSSMP, then so is the associated CSBP with immigration.

The first result in this paper, in Subsection 3.2, plays upon the explicit nature of all of the aforementioned processes and their special link with $\alpha$-stable processes, and specifies how we transform between each of them with the help of either a Doob $h$-transform or one of two possible space-time changes which are commonly referred to as Lamperti transformations. Some of these transformations are already known; however, we provide a complete picture. Our goal is then to take advantage of some of these transforms and to provide a more detailed description of the dynamics of both the self-similar CSBPs and the self-similar CSBPs with immigration.

Specifically, for self-similar CSBPs, we are interested in a more detailed description of how the process becomes extinct. We do this by time reversing its path from the moment of extinction. In particular, we specify integral tests furnishing law of the iterated logarithm (LIL) type results of the time-reversed process. For the case of self-similar CSBPs with immigration, we are interested in integral tests for its lower envelope at times 0 and $\infty$ when the process is issued from the origin. Understanding CSBPs and CSBPs with immigration in the context of self-similarity also leads to some explicit fluctuation identities involving their last passage times. This we do in Section 4. As our work is closely related to [17] in spirit, we conclude the paper with some remarks about a different kind of conditioning considered there which results in quasistationary distributions.

\section{Some processes revisited}

This section is dedicated to introducing more notation as well as providing more rigorous definitions of the stochastic processes that are of primary interest in this paper.

\subsection{Spectrally positive Lévy processes}

Let $\left(\mathrm{P}_{x}, x \in \mathbb{R}\right)$ be a family of probability measures on the space of càdlàg mappings (those that are continuous from the right with left limits) from $[0, \infty)$ to $\mathbb{R}$, denoted $\mathcal{D}$, such that, for each $x \in \mathbb{R}$, the canonical process $X$ is a Lévy process with no negative jumps issued from $x$. Set $\mathrm{P}:=\mathrm{P}_{0}$. So $\mathrm{P}_{x}$ is the law of $X+x$ under $\mathrm{P}$. The Laplace exponent $\psi:[0, \infty) \rightarrow(-\infty, \infty)$ of $X$ is specified by $\mathbb{E}\left(\exp \left\{-\lambda X_{t}\right\}\right)=\mathrm{e}^{t \psi(\lambda)}$ for $\lambda \in \mathbb{R}$, and can be expressed in the form

$$
\psi(\lambda)=a \lambda+\beta \lambda^{2}+\int_{(0, \infty)}\left(\mathrm{e}^{-\lambda x}-1+\lambda x \mathbf{1}_{\{x<1\}}\right) \Pi(\mathrm{d} x)
$$

where $a \in \mathbb{R}, \beta \geq 0$, and $\Pi$ is a $\sigma$-finite measure such that

$$
\int_{(0, \infty)}\left(1 \wedge x^{2}\right) \Pi(\mathrm{d} x)<\infty .
$$

Henceforth, we shall assume that $(X, \mathrm{P})$ is not a subordinator (recall that a subordinator is a Lévy process with increasing sample paths). In this case, it is known that the Laplace exponent $\psi$ is strictly convex and tends to $\infty$ as $\lambda$ goes to $\infty$. In this case, we define, for $q \geq 0$,

$$
\Phi(q)=\inf \{\lambda \geq 0: \psi(\lambda)>q\},
$$

the right inverse of $\psi$. Then $\Phi(0)$ is the largest root of the equation $\psi(\lambda)=0$. Theorem VII.1 of [1] implies that the condition $\Phi(0)>0$ holds if and only if the process drifts to $\infty$. Moreover, 
almost surely, the paths of $X$ drift to $\infty$, oscillate, or drift to $-\infty$ according respectively to whether $\psi^{\prime}(0+)<0, \psi^{\prime}(0+)=0$, or $\psi^{\prime}(0+)>0$.

\subsection{Conditioning to stay positive}

We also need to make use of Lévy processes conditioned to stay positive. The following commentary is taken from [1, Chapter VII] and [7], and is adapted to our setting, where $(X, \mathrm{P})$ is a Lévy process with no negative jumps, but not a subordinator. The process, $X$, conditioned to stay positive, is the strong Markov process whose law is given by

$$
\mathrm{P}_{x}^{\uparrow}\left(X_{t} \in \mathrm{d} y\right)=\lim _{q \downarrow 0} \mathrm{P}_{x}\left(X_{t} \in \mathrm{d} y, t<\frac{e}{q} \mid \tau_{0}>\frac{e}{q}\right), \quad t \geq 0, x, y>0,
$$

where $e$ is an independent and exponentially distributed random variable with mean 1 and

$$
\tau_{0}=\inf \left\{t>0: X_{t} \leq 0\right\} .
$$

It turns out that the measure on the left-hand side can also be constructed as the result of a Doob $h$-transform of $X$ killed when it first exits $(0, \infty)$. In the special case in which $\psi^{\prime}(0+) \leq 0$, the resulting semigroup is thus given by

$$
\mathrm{P}_{x}^{\uparrow}\left(X_{t} \in \mathrm{d} y\right)=\frac{1-\mathrm{e}^{-\Phi(0) y}}{1-\mathrm{e}^{-\Phi(0) x}} \mathrm{P}_{x}\left(X_{t} \in \mathrm{d} y, t<\tau_{0}\right), \quad t \geq 0, x, y>0,
$$

where the ratio on the right-hand side is understood as $y / x$ in the case in which $\Phi(0)=0$ (i.e. the case in which $\left.\psi^{\prime}(0+)=0\right)$. Moreover, the family of measures $\left(\mathrm{P}_{x}^{\uparrow}, x>0\right)$ induced on $\mathscr{D}$ are probability measures, and when $X$ has unbounded variation paths, the law $\mathrm{P}_{x}^{\uparrow}$ converges weakly as $x \downarrow 0$ to a measure denoted by $\mathrm{P}^{\uparrow}$.

Now, define $\widehat{X}:=-X$, the dual process of $X$. Denote by $\widehat{\mathrm{P}}_{x}$ the law of $\widehat{X}$ when issued from $x$ so that $\left(X, \widehat{\mathrm{P}}_{x}\right)=\left(\widehat{X}, \mathrm{P}_{-x}\right)$. The dual process conditioned to stay positive in the sense of (2) is again a Doob $h$-transform of $\left(X, \widehat{\mathrm{P}}_{x}\right)$ killed when it first exits $(0, \infty)$. In this case, assuming (conversely to $\mathrm{P}_{x}^{\uparrow}$ ) that $\psi^{\prime}(0+) \geq 0$, we have

$$
\widehat{\mathrm{P}}_{x}^{\uparrow}\left(X_{t} \in \mathrm{d} y\right)=\frac{W(y)}{W(x)} \widehat{\mathrm{P}}_{x}\left(X_{t} \in \mathrm{d} y, t<\tau_{0}\right), \quad t \geq 0, x, y>0,
$$

where $W$ is the so-called scale function for the process $-X$. The latter is the unique continuous function on $(0, \infty)$ with Laplace transform

$$
\int_{0}^{\infty} \mathrm{e}^{-\lambda x} W(x) \mathrm{d} x=\frac{1}{\psi(\lambda)}, \quad \lambda \geq 0,
$$

where we recall that $\psi$ is the Laplace exponent of $X$, defined in the previous subsection. In this case the measure $\widehat{\mathrm{P}}_{x}^{\uparrow}$ is always a probability measure, and there is always weak convergence as $x \downarrow 0$ to a probability measure, which we denote by $\widehat{\mathrm{P}}^{\uparrow}$.

\subsection{CSBPs and CSBPs with immigration}

CSBPs are the analogue of Bienaymé-Galton-Watson processes in continuous time and continuous-state space. Such classes of processes have been introduced by Jirina [14] and studied by many authors including Bingham [3], Grey [11], Grimvall [12], and Lamperti [18], [19], to name but a few. A CSBP $Y=\left(Y_{t}, t \geq 0\right)$ is a Markov process taking values in $[0, \infty]$, 
where 0 and $\infty$ are two absorbing states. Moreover, $Y$ satisfies the branching property; that is to say, the Laplace transform of $Y_{t}$ satisfies

$$
\mathbb{E}_{x}\left(\exp \left\{-\lambda Y_{t}\right\}\right)=\exp \left\{-x u_{t}(\lambda)\right\} \quad \text { for } \lambda \geq 0,
$$

for some function $u_{t}(\lambda)$. According to Silverstein [28], the function $u_{t}(\lambda)$ is determined by the integral equation

$$
\int_{u_{t}(\lambda)}^{\lambda} \frac{1}{\psi(u)} \mathrm{d} u=t
$$

where $\psi$ is the Laplace exponent of a spectrally positive Lévy process and is known as the branching mechanism of $Y$.

Lamperti [18] observed that CSBPs are connected to Lévy processes with no negative jumps by a simple time change. More precisely, consider the spectrally positive Lévy process $\left(X, \mathrm{P}_{x}\right)$ started at $x>0$ and with Laplace exponent $\psi$. Now, we introduce the clock

$$
A_{t}=\int_{0}^{t} \frac{\mathrm{d} s}{X_{s}}, \quad t \in\left[0, \tau_{0}\right),
$$

and its inverse

$$
\theta(t)=\inf \left\{s \geq 0: A_{s}>t\right\}
$$

Then the time-changed process $Y=\left(X_{\theta(t)}, t \geq 0\right)$, under $\mathrm{P}_{x}$, is a CSBP with initial population of size $x$. The transformation described above shall henceforth be referred to as the CSB Lamperti representation.

A CSBP is called supercritical, critical, or subcritical according respectively to whether its associated Lévy process drifts to $+\infty$, oscillates, or drifts to $-\infty$; in other words, according respectively to whether $\psi^{\prime}(0+)<0, \psi^{\prime}(0+)=0$, or $\psi^{\prime}(0+)>0$. It is known that a CSBP $Y$ with branching mechanism $\psi$ has a finite time extinction almost surely if and only if

$$
\int_{1}^{\infty} \frac{\mathrm{d} u}{\psi(u)}<\infty \quad \text { and } \quad \psi^{\prime}(0+) \geq 0 .
$$

In this work we are also interested in CSBPs with immigration. In the remainder of this subsection we assume that the CSBP is critical, i.e. $\psi^{\prime}(0+)=0$. Recall that a CSBP with immigration is a strong Markov process taking values in $[0, \infty]$, where 0 is no longer absorbing. If $\left(Y_{t}^{\uparrow}: t \geq 0\right)$ is a process in this class then its semigroup is characterised by

$$
\mathbb{E}_{x}\left(\exp \left\{-\lambda Y_{t}^{\uparrow}\right\}\right)=\exp \left\{-x u_{t}(\lambda)-\int_{0}^{t} \phi\left(u_{t-s}(\lambda)\right) \mathrm{d} s\right\} \quad \text { for } \lambda \geq 0,
$$

where $\phi$ is a Bernstein function satisfying $\phi(0)=0$ and is referred to as the immigration mechanism. See, for example, [17] for a formal definition. Roelly and Rouault [26], and more recently Lambert [17], showed that if

$$
T_{0}=\inf \left\{t>0: Y_{t}=0\right\}
$$

then the limit

$$
\lim _{s \uparrow \infty} \mathrm{P}_{x}\left(Y_{t} \in \mathrm{d} y \mid T_{0}>t+s\right), \quad t \geq 0, x, y>0
$$


exists and defines a semigroup which is that of a CSBP with immigration having initial population size $x$ and immigration mechanism

$$
\phi(\lambda)=\psi^{\prime}(\lambda), \quad \lambda \geq 0 .
$$

The limit (7) may be thought of as conditioning the CSBP not to become extinct.

Lambert [17] also proved an interesting connection between the conditioning for a CSBP (7) and for the underlying Lévy process (2). Specifically, Lambert showed that $\left(X_{\theta}, \mathrm{P}_{x}^{\uparrow}\right)=$ $\left(Y^{\uparrow}, \mathrm{P}_{x}\right)$, where the latter process has an immigration mechanism given by $\psi^{\prime}(\lambda)$. Another way of phrasing this is that the CSBP with immigration obtained by conditioning a critical CSBP not to become extinct is equal in law to the underlying spectrally positive Lévy process conditioned to stay positive and then time changed with the CSB Lamperti representation. Moreover, Lambert also showed that, when $\mathrm{P}_{x}^{\uparrow}$ is used to describe the law of $Y$, then it fulfills the following Doob $h$-transform:

$$
\mathrm{P}_{x}^{\uparrow}\left(Y_{t} \in \mathrm{d} y\right)=\frac{y}{x} \mathrm{P}_{x}\left(Y_{t} \in \mathrm{d} y, t<T_{0}\right) \quad \text { for } y>0 \text { and } t \geq 0 .
$$

\section{Self-similarity, and $\alpha$-stable CSBPs and $\alpha$-stable CSBPs with immigration}

Before we investigate self-similar CSBPs and self-similar CSBPs with immigration, let us first address how self-similarity and the associated Lamperti transformation manifests itself for the case of a spectrally positive $\alpha$-stable process. This turns out to be key to understanding self-similarity of CSBPs and CSBPs with immigration.

\subsection{Stable processes and the PSSMP Lamperti representation}

Stable Lévy processes with no negative jumps are Lévy processes with Laplace exponent of the type given in (1) that satisfy the scaling property for some index $\alpha>0$. More precisely, there exists a constant $\alpha>0$ such that, for any $k>0$,

$$
\text { the law of }\left(k X_{k^{-\alpha} t}, t \geq 0\right) \text { under } \mathrm{P}_{x} \text { is } \mathrm{P}_{k x} .
$$

In this subsection, $\left(X, \mathrm{P}_{x}\right)$ shall denote a stable Lévy process with no negative jumps of index $\alpha \in(1,2]$ starting at $x \in \mathbb{R}$ (see Chapter VII of [1] for further discussion on stable Lévy processes). It is known that the Laplace exponent of $\left(X, \mathrm{P}_{x}\right)$ takes the form

$$
\psi(\lambda)=c_{+} \lambda^{\alpha}, \quad \lambda \geq 0, \alpha \in(0,2),
$$

where $c_{+}$is a nonnegative constant. The case in which $\alpha=2$ corresponds to the process $\left(X, \mathrm{P}_{x}\right)$ being a multiple of standard Brownian motion. In the remainder of this work, when we consider the case in which $\alpha=2$, we shall refer to the Brownian motion, i.e. we choose $c_{+}=\frac{1}{2}$.

Recall that the stable Lévy process killed at the first time that it enters the negative half-line is defined by

$$
X_{t}^{\dagger}:=X_{t} \mathbf{1}_{\left\{t<\tau_{0}\right\}}, \quad t \geq 0,
$$

where $\tau_{0}=\inf \left\{t \geq 0: X_{t} \leq 0\right\}$. From the previous subsection, a stable Lévy process with no negative jumps conditioned to stay positive is tantamount to a Doob- $h$ transform of the killed process where $h(x)=x$. According to Caballero and Chaumont [4], both the process $X$ and its conditioned version belong to the class of PSSMPs; that is to say, positive Markov processes satisfying property (8). 
From Lamperti's [19] work, it is known that the family of PSSMPs up to its first hitting time of 0 may be expressed as the exponential of a Lévy process, time changed by the inverse of its exponential functional. More precisely, let $\left(X, \mathrm{Q}_{x}\right)$ be a self-similar Markov process started from $x>0$ that fulfills the scaling property for some $\alpha>0$. Then, under $\mathrm{Q}_{x}$, there exists a Lévy process $\xi=\left(\xi_{t}, t \geq 0\right)$, possibly killed at an independent exponential time, which does not depend on $x$ and such that

$$
X_{t}=x \exp \left\{\xi_{\zeta\left(t x^{-\alpha}\right)}\right\}, \quad 0 \leq t \leq x^{\alpha} I(\alpha \xi),
$$

where

$$
\begin{gathered}
\zeta(t)=\inf \left\{s \geq 0: I_{s}(\alpha \xi)>t\right\}, \\
I_{s}(\alpha \xi)=\int_{0}^{s} \exp \left\{\alpha \xi_{u}\right\} \mathrm{d} u, \\
\text { and } \quad I(\alpha \xi)=\lim _{t \rightarrow+\infty} I_{t}(\alpha \xi)
\end{gathered}
$$

We shall refer to this transformation as the PSSMP Lamperti representation.

In [9], it was shown that a stable Lévy process with index $\alpha \in(1,2)$ killed at the first time that it enters the negative half-line has underlying Lévy process $\xi$, whose Laplace exponent is given by

$$
\Psi(\lambda)=m \frac{\Gamma(\lambda+\alpha)}{\Gamma(\lambda) \Gamma(\alpha)} \quad \text { for } \lambda \geq 0,
$$

where $m>0$ is the mean of $-\xi$, which is finite. Note that this last fact implies that the process $\xi$ drifts towards $-\infty$. In the Brownian case, i.e. when $\alpha=2$, the Lévy process $\xi$ is a Brownian motion with drift $a=-\frac{1}{2}$.

The Laplace exponent of the underlying Lévy process, denoted by $\xi^{*}$, of the stable Lévy process (with $\alpha \in(1,2)$ ) conditioned to stay positive is also computed in [9]. It is given by

$$
\Psi^{*}(\lambda)=m \frac{\Gamma(\lambda-1+\alpha)}{\Gamma(\lambda-1) \Gamma(\alpha)} \quad \text { for } \lambda \geq 0
$$

Here we use the gamma function for values $x \in(-1,0)$ via the relation $x \Gamma(x)=\Gamma(1+x)$. In this case, the Lévy process $\xi^{*}$ drifts towards $+\infty$. When $\alpha=2$, it is not difficult to show that the process $\xi^{*}$ is a Brownian motion with drift $a=\frac{1}{2}$.

Next we remark that, under $\widehat{\mathrm{P}}_{x}$, the stable Lévy process $X$ has no positive jumps. From Corollary 6 of [9], it is known that the underlying Levy process in the PSSMP Lamperti representation of the spectrally negative stable Lévy process conditioned to stay positive is $\hat{\xi}$, the dual of $\xi$. Note that in the case in which $\alpha=2$, the processes $\hat{\xi}$ and $\xi^{*}$ are the same.

There is a definitive relation between $\xi$ and $\xi^{*}$ which shall be used later and, hence, we register it as a proposition below. Its proof can be found in [9]. In the sequel, Pr shall be a reference probability measure on $\mathscr{D}$ (with associated expectation operator $\mathrm{E}$ ) under which $\xi$ and $\xi^{*}$ are Lévy processes, issued from the origin, whose respective laws are defined above.

Proposition 1. For every $t \geq 0$ and every bounded measurable function $f$,

$$
\mathrm{E}\left(f\left(\xi_{t}^{*}\right)\right)=\mathrm{E}\left(\exp \left\{\xi_{t}\right\} f\left(\xi_{t}\right)\right) .
$$

In particular, the process $-\xi^{*}$ and $\xi$ satisfy Cramér's condition, i.e.

$$
\mathrm{E}\left(\exp \left\{-\xi_{1}^{*}\right\}\right)=1 \quad \text { and } \quad \mathrm{E}\left(\exp \left\{\xi_{1}\right\}\right)=1 .
$$


Finally, we note that $\xi$ and $\xi^{*}$ are two examples of so-called Lamperti stable processes (see, for instance, [2], [4], [6], [10], and [24] for related expositions and the formal definition of a Lamperti stable process).

\subsection{PSSMP Lamperti representation for CSBPs and CSBPs with immigration}

Suppose, as in the previous section, that $\left(X, \mathrm{P}_{x}\right)$ is a spectrally positive $\alpha$-stable process with index $\alpha \in(1,2]$ starting from $x>0$. We refer to $Y$, the associated CSBP, as the $\alpha$-stable CSBP. Moreover, when talking of the latter process conditioned to stay positive in the sense of the description in Subsection 2.3, i.e. the processes $\left(Y, \mathrm{P}_{x}^{\uparrow}\right)$ for $x>0$, we shall refer to the associated CSBP with immigration as the $\alpha$-stable CSBP with immigration.

We begin by showing that $\alpha$-stable CSBPs and $\alpha$-stable CSBPs with immigration are selfsimilar processes (positivity is obvious). To this end, we state and prove a generic result which, in some sense, is well-known folklore and shall be useful throughout the remainder of this section. For the sake of completeness, we include its proof.

Proposition 2. Suppose that $X$ is any PSSMP issued from $x>0$ with self-similarity index $\alpha>1$, and let $\theta$ be the CSB Lamperti time change. Then $X_{\theta}$ is a PSSMP issued from $x$ with self-similarity index $\alpha-1$ and with the same underlying Lévy process as $X$.

Proof. Suppose that $\eta$ is the underlying Lévy process for the process $X$. We first define

$$
A .=\int_{0}^{\cdot} \frac{\mathrm{d} s}{X_{s}}, \quad I .(\alpha \eta)=\int_{0}^{.} \exp \left\{\alpha \eta_{s}\right\} \mathrm{d} s, \quad \text { and } \quad I .((\alpha-1) \eta)=\int_{0}^{.} \exp \left\{(\alpha-1) \eta_{s}\right\} \mathrm{d} s .
$$

Recall that $\zeta(\cdot)$ is the right-continuous inverse of $I .(\alpha \eta)$. From the PSSMP Lamperti transform of $X$ and the change of variable $s=x^{\alpha} I_{u}(\alpha \eta)$, we obtain

$$
\begin{aligned}
A_{x^{\alpha} I .(\alpha \eta)} & =\int_{0}^{x^{\alpha} I .(\alpha \eta)} \frac{\mathrm{d} s}{x \exp \left\{\eta_{\zeta\left(s / x^{\alpha}\right)}\right.} \\
& =x^{\alpha-1} \int_{0}^{\cdot} \frac{\exp \left\{\alpha \eta_{u}\right\}}{\exp \left\{\eta_{u}\right\}} \mathrm{d} u \\
& =x^{\alpha-1} \int_{0}^{\cdot} \exp \left\{(\alpha-1) \eta_{u}\right\} \mathrm{d} u .
\end{aligned}
$$

On the other hand, the right-continuous inverse of $I((\alpha-1) \eta)$ is defined by

$$
h(t)=\inf \left\{s \geq 0: I_{s}((\alpha-1) \eta)>t\right\},
$$

and recall that $\theta$ is the right-continuous inverse function of $A$. Hence, we have, for any $0 \leq t<x^{\alpha-1} I_{\infty}((\alpha-1) \eta)$

$$
\begin{aligned}
h\left(\frac{t}{x^{\alpha-1}}\right) & =\inf \left\{s \geq 0: I_{s}((\alpha-1) \eta)>\frac{t}{x^{\alpha-1}}\right\} \\
& =\inf \left\{s \geq 0: A_{x^{\alpha}} I_{s}(\alpha \eta)>t\right\} \\
& =\inf \left\{\zeta\left(\frac{u}{x^{\alpha}}\right) \geq 0: A_{u}>t\right\} \\
& =\zeta\left(\frac{\theta(t)}{x^{\alpha}}\right) .
\end{aligned}
$$


From the PSSMP Lamperti representation of $X$, we have

$$
\inf \left\{t \geq 0: X_{\theta(t)}=0\right\}=x^{\alpha-1} I_{\infty}((\alpha-1) \eta)
$$

and, for all $0 \leq t \leq x^{\alpha-1} I_{\infty}((\alpha-1) \eta)$,

$$
X_{\theta(t)}=x \exp \left\{\eta_{\zeta\left(\theta(t) / x^{\alpha}\right)}\right\}=x \exp \left\{\eta_{h\left(t / x^{\alpha-1}\right)}\right\} .
$$

This completes the proof.

This leads immediately to the conclusion that the $\alpha$-stable CSBPs and the $\alpha$-stable CSBPs with immigration are self-similar with index $\alpha-1$.

Corollary 1. (i) The process $\left(Y, \mathrm{P}_{x}\right)$ is a PSSMP with self-similarity index $\alpha-1$. Moreover, its PSSMP Lamperti representation under $\mathrm{P}_{x}$ is given by

$$
Y_{t}=x \exp \left\{\xi_{h\left(t x^{-(\alpha-1)}\right)}\right\}, \quad 0 \leq t \leq x^{\alpha-1} \int_{0}^{\infty} \exp \left\{(\alpha-1) \xi_{u}\right\} \mathrm{d} u,
$$

where

$$
h(t)=\inf \left\{s \geq 0: \int_{0}^{s} \exp \left\{(\alpha-1) \xi_{u}\right\} \mathrm{d} u>t\right\} .
$$

(ii) The process $\left(Y, \mathrm{P}_{x}^{\uparrow}\right)$ is a PSSMP with self-similarity index $\alpha-1$. Moreover, its PSSMP Lamperti representation under $\mathrm{P}_{x}^{\uparrow}$ is given by

$$
Y_{t}=x \exp \left\{\xi_{\zeta^{*}\left(t x^{-(\alpha-1)}\right)}^{*}\right\}, \quad t \geq 0,
$$

where

$$
\zeta^{*}(t)=\inf \left\{s \geq 0: \int_{0}^{s} \exp \left\{(\alpha-1) \xi_{u}^{*}\right\} \mathrm{d} u>t\right\} .
$$

Corollary 1 , Proposition 1 , and the discussion in the previous sections give rise to the flow of transformations given in Figure 1, where

$$
h_{1}(y)=\mathrm{e}^{y}, \quad h_{2}(y)=\mathrm{e}^{-y}, \quad h_{3}(y)=y, \quad \text { and } \quad h_{4}=\frac{1}{y} .
$$

The vertical arrows in Figure 1 are the result of a Doob $h$-transform with the $h$-function indicated in each direction, and the parameters $\alpha$ and $\alpha-1$ are the index of self-similarity on the PSSMP Lamperti representation.

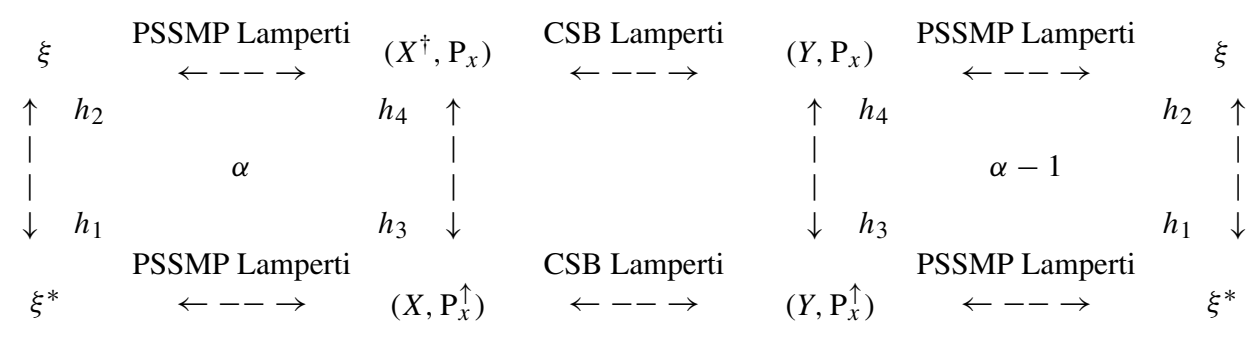

FIGURE 1. 


\section{Some path properties of $\alpha$-stable CSBPs and $\alpha$-stable CSBPs with immigration}

In this section we state the remainder of our main results, leaving the proofs to the next section. Throughout, we shall always assume that $X$ is a spectrally positive $\alpha$-stable process unless otherwise stated.

\subsection{Entrance laws}

We begin by introducing

$$
\sigma_{x}=\sup \left\{t>0: X_{t} \leq x\right\},
$$

the last passage time of $X$ below $x \in \mathbb{R}$, for Theorem 1, below, which gives us self-similarity of the $\alpha$-stable CSBP when time reversed from extinction. Recall the notation $\widehat{\mathrm{P}}^{\uparrow}$ for the law of $\widehat{X}$ conditioned to stay positive. We remark that, under $\widehat{\mathrm{P}}^{\uparrow}$, the canonical process $X$ drifts towards $\infty$ and also that $X_{t}>0$ for $t>0$.

Theorem 1. For each $x>0$,

$$
\left\{\left(Y_{\left(T_{0}-t\right)-}: t<T_{0}\right), \mathrm{P}_{x}\right\} \stackrel{\mathrm{D}}{=}\left\{\left(X_{\theta(t)}, 0 \leq t<A_{\sigma_{x}}\right), \widehat{\mathrm{P}}^{\uparrow}\right\}
$$

where $\stackrel{\text { D }}{=}$ ' denotes equality in law or distribution. Moreover, the process $X_{\theta}:=\left(X_{\theta(t)}, t \geq 0\right)$ under $\widehat{\mathrm{P}}^{\uparrow}$ is a PSSMP with index $\alpha-1$, starting from 0 , with the same semigroup as the processes $\left(X_{\theta}, \widehat{\mathrm{P}}_{y}^{\uparrow}\right)$ for $y>0$, and with entrance law given by

$$
\widehat{\mathbb{E}}^{\uparrow}\left(f\left(X_{\theta(t)}\right)\right)=\frac{c_{\alpha}}{m} \int_{0}^{\infty} f\left(t^{1 /(\alpha-1)} x\right) x^{\alpha-1} \exp \left\{-c_{\alpha} x\right\} \mathrm{d} x,
$$

where $c_{\alpha}=\left(c_{+}(\alpha-1)\right)^{-1 /(\alpha-1)}, t>0$, and $f$ is a positive measurable function.

Remark 1. It is important to note that, when $\alpha=2$, the process $\left(X_{\theta(t)}, t \geq 0\right)$ under $\widehat{\mathrm{P}}^{\uparrow}$ is in fact the CSBP with immigration. This follows from the remark made in Subsection 3.1 that in this particular case we have $\hat{\xi}=\xi^{*}$.

Remark 2. As we shall see in the next section when we prove this theorem, the identity in law (9) is in fact true for any CSBP (not just $\alpha$-stable CSBPs) which becomes extinct almost surely (and, thus, the conditions in (6) are in force).

In the case of an $\alpha$-stable CSBP with immigration we have thus far only discussed the case in which it is issued from $x>0$. The following theorem tells us that the $\alpha$-stable CSBP with immigration is still well defined as a self-similar process when issued from the origin. Moreover, the theorem also specifies the entrance law.

Theorem 2. The process $\left(Y, \mathrm{P}_{x}^{\uparrow}\right)$ converges weakly with respect to the Skorokhod topology as $x$ tends to 0 towards $\left(Y, \mathrm{P}^{\uparrow}\right)$, a PSSMP starting from 0 with the same semigroup as $\left(Y, \mathrm{P}_{x}^{\uparrow}\right)$ for $x>0$, and with entrance law given by

$$
\mathbb{E}^{\uparrow}\left(\exp \left\{-\lambda Y_{t}\right\}\right)=\left(1+c_{+}(\alpha-1) t \lambda^{\alpha-1}\right)^{-\alpha /(\alpha-1)} .
$$

\subsection{Asymptotic results}

Theorems 3, 4, and 5, below, each study the asymptotic behaviour for the $\alpha$-stable CSBP towards its moment of extinction. We start by stating the integral test for the lower envelope of $\left(Y_{\left(T_{0}-t\right)^{-}}, 0 \leq t \leq T_{0}\right)$ under $\mathrm{P}_{x}$ at 0 . 
Theorem 3. Let $f$ be an increasing function such that $\lim _{t \rightarrow 0} f(t) / t=0$. Then, for every $x>0$,

$$
\mathrm{P}_{x}\left(Y_{\left(T_{0}-t\right)^{-}}<f^{1 /(\alpha-1)}(t) \text { infinitely often (i.o.) as } t \rightarrow 0\right)=0 \text { or } 1
$$

according respectively to whether

$$
\int_{0+} f^{1 /(\alpha-1)}(t) t^{-\alpha /(\alpha-1)} \mathrm{d} t
$$

is finite or infinite. In particular,

$$
\liminf _{t \rightarrow 0} \frac{Y_{\left(T_{0}-t\right)^{-}}}{t^{\kappa}}=\left\{\begin{array}{ll}
0 & \text { if } \kappa<1 /(\alpha-1), \\
+\infty & \text { if } \kappa \geq 1 /(\alpha-1),
\end{array} \quad \mathrm{P}_{x} \text {-almost surely }\left(\mathrm{P}_{x} \text {-a.s. }\right) .\right.
$$

Next, introduce $\mathscr{H}_{0}$, the class of increasing functions $f:(0,+\infty) \mapsto[0,+\infty)$ such that

(i) $f(0)=0$ and

(ii) there exists a $\beta \in(0,1)$ such that $\sup _{t<\beta} t / f(t)<\infty$.

We also denote by $\underline{Y}_{t}$ the infimum of the $\operatorname{CSBP}\left(Y, \mathrm{P}_{x}\right)$ over $[0, t]$. The upper envelope of the process $\left(\underline{Y}_{\left(T_{0}-t\right)^{-}}, 0 \leq t \leq T_{0}\right)$ under $\mathrm{P}_{x}$ at 0 is described by the integral test in the following theorem.

Theorem 4. Let $f \in \mathscr{H}_{0}$. Then the following statements hold.

(i) If

$$
\int_{0+} \exp \left\{-\left(\frac{c_{+}(\alpha-1) t}{f(t)}\right)^{-1 /(\alpha-1)}\right\} \frac{\mathrm{d} t}{t}<\infty
$$

then, for all $\varepsilon, x>0$,

$$
\mathrm{P}_{x}\left(\underline{Y}_{\left(T_{0}-t\right)^{-}}>(1+\varepsilon) f^{1 /(\alpha-1)}(t) \text { i.o. as } t \rightarrow 0\right)=0 .
$$

(ii) If

$$
\int_{0+} \exp \left\{-\left(\frac{c_{+}(\alpha-1) t}{f(t)}\right)^{-1 /(\alpha-1)}\right\} \frac{\mathrm{d} t}{t}=\infty
$$

then, for all $\varepsilon, x>0$,

$$
\mathrm{P}_{x}\left(\underline{Y}_{\left(T_{0}-t\right)^{-}}>(1-\varepsilon) f^{1 / \alpha-1}(t) \text { i.o. as } t \rightarrow 0\right)=1 .
$$

In particular, we have the following LIL:

$$
\limsup _{t \rightarrow 0} \frac{\underline{Y}_{\left(T_{0}-t\right)^{-}}}{t^{1 /(\alpha-1)}(\log \log (1 / t))^{1-\alpha}}=\left(c_{+}(\alpha-1)\right)^{1 /(\alpha-1)} \quad \mathrm{P}_{x} \text {-a.s. }
$$

Finally, still within the framework of $\alpha$-stable CSBPs, the following result describes the upper envelope of the time-reversed processes $\left(Y_{\left(T_{0}-t\right)^{-}}, 0 \leq t \leq T_{0}\right)$ and $\left((Y-\underline{Y})_{\left(T_{0}-t\right)^{-}}, 0 \leq t \leq\right.$ $T_{0}$ ), in the form of a LIL. 
Theorem 5. For every $x>0$, we have

$$
\limsup _{t \rightarrow 0} \frac{Y_{\left(T_{0}-t\right)^{-}}}{t^{1 /(\alpha-1)}(\log \log (1 / t))^{1-\alpha}}=\left(c_{+}(\alpha-1)\right)^{1 /(\alpha-1)} \quad \mathrm{P}_{x} \text {-a.s. }
$$

and

$$
\limsup _{t \rightarrow 0} \frac{(Y-\underline{Y})_{\left(T_{0}-t\right)^{-}}}{t^{1 /(\alpha-1)}(\log \log (1 / t))^{1-\alpha}}=\left(c_{+}(\alpha-1)\right)^{1 /(\alpha-1)} \quad \mathrm{P}_{x} \text {-a.s. }
$$

We should note that, when $\alpha=2$, the upper envelope of the time-reversed process $\left\{\left(Y_{\left(T_{0}-t\right)^{-}}\right.\right.$, $\left.\left.0 \leq t \leq T_{0}\right), \mathrm{P}_{x}\right\}$ is described by the Kolmogorov-Dvoretsky-Erdős integral test, since the latter process has the same law as the square of a Bessel process of dimension $d=3$ killed at its last passage time below the level $x>0$. (See, for instance, [13].)

Next we turn to the integral test which describes the lower envelope at 0 and at $\infty$ of the $\alpha$-stable CSBP with immigration.

Theorem 6. Let $f$ be an increasing function such that $\lim _{t \rightarrow 0} f(t) / t=0$. Then

$$
\mathrm{P}^{\uparrow}\left(Y_{t}<f^{1 /(\alpha-1)}(t) \text { i.o. as } t \rightarrow 0\right)=0 \text { or } 1
$$

according respectively to whether

$$
\int_{0+} f^{1 /(\alpha-1)}(t) t^{-\alpha /(\alpha-1)} \mathrm{d} t
$$

is finite or infinite. In particular,

$$
\liminf _{t \rightarrow 0} \frac{Y_{t}}{t^{\kappa}}=\left\{\begin{array}{ll}
0 & \text { if } \kappa<1 /(\alpha-1), \\
+\infty & \text { if } \kappa \geq 1 /(\alpha-1),
\end{array} \quad \mathrm{P}^{\uparrow}\right. \text {-a.s. }
$$

Let $g$ be an increasing function such that $\lim _{n \rightarrow \infty} g(t) / t=0$. Then, for all $x \geq 0$,

$$
\mathrm{P}_{x}^{\uparrow}\left(Y_{t}<g^{1 /(\alpha-1)}(t) \text { i.o. as } t \rightarrow \infty\right)=0 \text { or } 1
$$

according respectively to whether

$$
\int^{\infty} g^{1 /(\alpha-1)}(t) t^{-\alpha /(\alpha-1)} \mathrm{d} t
$$

is finite or infinite. In particular, for any $x \geq 0$,

$$
\liminf _{t \rightarrow \infty} \frac{Y_{t}}{t^{\kappa}}=\left\{\begin{array}{ll}
0 & \text { if } \kappa \geq 1 /(\alpha-1), \\
+\infty & \text { if } \kappa<1 /(\alpha-1),
\end{array} \quad \mathrm{P}_{x}\right. \text {-a.s. }
$$

\subsection{Fluctuation identities at last passage}

Define the last passage time above $x$ of $\xi$ by

$$
D_{x}=\sup \left\{t \geq 0: \xi_{t} \geq x\right\} .
$$


Theorem 7. Suppose that the Lévy process $(X, \mathrm{P})$ does not drift towards $+\infty$. Then, for every $x>0$ and $0<y \leq x$,

$$
\mathrm{P}_{x}\left(\inf _{0 \leq t \leq U_{y}} Y_{t} \geq z\right)=\frac{W(y-z)}{W(y)} \mathbf{1}_{\{z \leq y\}},
$$

where $U_{y}=\sup \left\{t>0: Y_{t} \geq y\right\}$ and the scale function $W$ satisfies (3). In particular, in the case in which $X$ is a spectrally positive $\alpha$-stable process with $\alpha \in(1,2]$,

$$
\mathrm{P}_{x}\left(\inf _{0 \leq t \leq U_{y}} Y_{t} \geq z\right)=\operatorname{Pr}\left(\inf _{0 \leq t \leq D_{u}} \xi_{t} \geq v\right)=\left(1-\mathrm{e}^{v-u}\right)^{\alpha-1},
$$

where $v=\log (z / x), u=\log (y / x)$, and $z \leq y$.

Next we establish a similar result, but for the supremum at last passage for the CSBP with immigration $\left(Y, \mathrm{P}^{\uparrow}\right)$. For this, we define

$$
U_{y}^{-}=\sup \left\{t \geq 0: Y_{t} \leq y\right\} .
$$

Theorem 8. Let $z \geq y>0$. Then

$$
\mathrm{P}^{\uparrow}\left(\sup _{0 \leq s<U_{y}^{-}} Y_{s} \leq z\right)=1-\frac{y}{m^{*} z},
$$

where $m^{*}$ is the mean of $\xi_{1}^{*}$.

Now we define the following exponential functional of $\xi^{*}$ :

$$
I^{*}:=\int_{0}^{\infty} \exp \left\{-\alpha \xi_{s}^{*}\right\} \mathrm{d} s .
$$

The exponential functional $I^{*}$ was studied by Chaumont et al. [9]. In particular, Chaumont $e t$ al. [9] found that

$$
\operatorname{Pr}\left(\frac{1}{I^{*}} \in \mathrm{d} y\right)=\alpha m^{*} q_{1}(y) \mathrm{d} y,
$$

where $q_{1}$ is the density of the entrance law of the excursion measure under $\mathrm{P}$ of the reflected process, $\left(X_{t}-\underline{X}_{t}, t \geq 0\right)$, away from 0 .

The time-reversal property of $\left(Y, \mathrm{P}^{\uparrow}\right)$ at its last passage time (see Proposition 1 of [8]) combined with the CSB Lamperti representation and the PSSMP Lamperti representation gives us the following result for the total progeny of the self-similar CSBP with immigration.

Proposition 3. For $y>0$, the total progeny of $\left(Y, \mathrm{P}^{\uparrow}\right)$ up to time $U_{y}^{-}, \int_{0}^{U_{y}^{-}} Y_{s} \mathrm{~d} s$, and the last passage time of $\left(X, \mathrm{P}^{\uparrow}\right)$ below $y, \sigma_{y}$, are both equal in law to $\Gamma^{\alpha} I^{*}$, where $\Gamma=Y_{U_{y}^{-}}$and is independent of $I^{*}$.

\section{Proofs}

\subsection{Proofs of Theorems 1 and 2}

We start by establishing some preliminary results needed for the proof of Theorem 1 .

Proposition 4. Suppose that $X$ is any spectrally positive Lévy process. If the conditions in (6) are satisfied then, for every $y>0$,

$$
\left\{\left(Y_{\left(T_{0}-t\right)-}, 0 \leq t<T_{0}\right), \mathrm{P}_{y}\right\} \stackrel{\mathrm{D}}{=}\left\{\left(X_{\theta(t)}, 0 \leq t<A_{\sigma_{y}}\right), \widehat{\mathrm{P}}^{\uparrow}\right\} .
$$


Proof. We first prove that the CSB Lamperti representation is well defined for the process $\left(X, \widehat{\mathrm{P}}^{\uparrow}\right)$. In order to do this, it is enough to prove that the map $s \mapsto 1 / X_{s}$ is $\widehat{\mathrm{P}}^{\uparrow}$-a.s. integrable in a neighbourhood of 0 . To this end, recall that (6) implies that $\mathrm{P}_{y}\left(\tau_{0}<\infty\right)=1$ for all $y \geq 0$. Hence, by Theorem VII.18 of [1], we know that, for $y>0$,

$$
\left\{\left(X_{t}, 0 \leq t<\sigma_{y}\right), \widehat{\mathrm{P}}^{\uparrow}\right\} \stackrel{\mathrm{D}}{=}\left\{\left(X_{\left(\tau_{0}-t\right)-}, 0 \leq t<\tau_{0}\right), \mathrm{P}_{y}\right\},
$$

which in turn can be used to deduce that

$$
\int_{0}^{\sigma_{y}} \frac{1}{X_{s}} \mathrm{~d} s \text { under } \widehat{\mathrm{P}}^{\uparrow} \text { is equal in law to } \int_{0}^{\tau_{0}} \frac{1}{X_{s}} \mathrm{~d} s \text { under } \mathrm{P}_{y} .
$$

However, the latter integral is equal to $T_{0}$, which is $\mathrm{P}_{y}$-a.s. finite given the assumptions in (6).

Next, from the definition of $Y$, under $\mathrm{P}_{y}$, we have

$$
\left(Y_{\left(T_{0}-t\right)-}, 0 \leq t<T_{0}\right)=\left(X_{\theta\left(A_{\tau_{0}}-t\right)-}, 0 \leq t<A_{\tau_{0}}\right) .
$$

Define

$$
\theta^{\prime}(t)=\inf \left\{s>0: B_{s}>t\right\} \quad \text { where } \quad B_{s}=\int_{0}^{s} \frac{1}{X_{\tau_{0}-u}} \mathrm{~d} u .
$$

Setting $t=B_{s}$, we have

$$
A_{\tau_{0}}-B_{s}=\int_{0}^{\tau_{0}} \frac{1}{X_{u}} \mathrm{~d} u-\int_{0}^{s} \frac{1}{X_{\tau_{0}-u}} \mathrm{~d} u=\int_{0}^{\tau_{0}-s} \frac{1}{X_{u}} \mathrm{~d} u
$$

and, hence,

$$
X_{\theta\left(A_{\tau_{0}}-t\right)-}=X_{\theta\left(A_{\tau_{0}-s}\right)-}=X_{\left(\tau_{0}-s\right)-}=X_{\left(\tau_{0}-\theta^{\prime}(t)\right)-} .
$$

As noted earlier, $T_{0}=A_{\tau_{0}}=B_{\tau_{0}}$. Now it follows, from (14), (15), and (16), that

$$
\left\{\left(Y_{\left(T_{0}-t\right)-}, 0 \leq t<T_{0}\right), \mathrm{P}_{y}\right\} \stackrel{\mathrm{D}}{=}\left\{\left(X_{\theta(t)}, 0 \leq t<A_{\sigma_{y}}\right), \widehat{\mathrm{P}}^{\uparrow}\right\}
$$

as required.

We now need the following auxiliary lemma which says in particular that the distribution of $I_{\infty}((\alpha-1) \xi)=\int_{0}^{\infty} \exp \left\{(\alpha-1) \xi_{t}\right\} \mathrm{d} t$ has a Fréchet distribution and, moreover, that the Fréchet distribution is self-decomposable.

Lemma 1. The distribution of $I:=I_{\infty}((\alpha-1) \xi)$ is given by

$$
\operatorname{Pr}(I \leq t)=\exp \left\{-\left(c_{+}(\alpha-1) t\right)^{-1 /(\alpha-1)}\right\}
$$

Moreover, I is self-decomposable and, thus, has a completely monotone density with respect to the Lebesgue measure.

Proof. From the PSSMP Lamperti representation of $\left(Y, \mathrm{P}_{x}\right)$ and Proposition 4, we deduce that $T_{0}=x^{\alpha-1} I$. From [3], it is known that

$$
\mathrm{P}_{x}\left(T_{0} \leq t\right)=\exp \left\{-x u_{t}(\infty)\right\}
$$

where $u_{t}(\infty)$ solves

$$
\int_{u_{t}(\infty)}^{\infty} \frac{1}{c_{+} v^{\alpha}} \mathrm{d} v=t
$$


Therefore, $u_{t}(\infty)=\left(c_{+}(\alpha-1) t\right)^{-1 /(\alpha-1)}$ and, hence,

$$
\mathrm{P}_{x}\left(T_{0} \leq t\right)=\operatorname{Pr}\left(I \leq \frac{t}{x^{\alpha-1}}\right)=\exp \left\{-x\left[c_{+}(\alpha-1) t\right]^{-1 /(\alpha-1)}\right\}
$$

which implies (17).

Let $a<0$. Then

$$
I=\int_{0}^{\infty} \exp \left\{(\alpha-1) \xi_{u}\right\} \mathrm{d} u=\int_{0}^{S_{a}} \exp \left\{(\alpha-1) \xi_{u}\right\} \mathrm{d} u+\mathrm{e}^{(\alpha-1) a} \int_{0}^{\infty} \exp \left\{(\alpha-1) \xi_{u}^{\prime}\right\} \mathrm{d} u,
$$

where $\xi^{\prime}=\left(\xi_{S_{a}+t}-a, t \geq 0\right)$ and $S_{a}=\inf \left\{t \geq 0: \xi_{t} \leq a\right\}$. Then self-decomposability follows from the independence of $\left(\xi_{t}, 0 \leq t \leq \tau_{a}\right)$ and $\xi^{\prime}$. Self-decomposable distributions on $\mathbb{R}_{+}$are unimodal (see, for instance, Chapter 10 of [27]), i.e. they have a completely monotone density on $(0, \infty)$, with respect to the Lebesgue measure.

Proof of Theorem 1. The time-reversal property, (9), follows from Proposition 4. The PSSMP Lamperti representation of the process $\left(X_{\theta(t)}, t \geq 0\right)$ under $\widehat{\mathrm{P}}_{y}^{\uparrow}$ when issued from $y>0$ follows from Proposition 2, noting in particular that $\left(X, \widehat{\mathrm{P}}_{y}^{\uparrow}\right)$ is a spectrally positive stable process conditioned to stay positive, which is a positive, self-similar process with index $\alpha$.

The Lévy process $\hat{\xi}$ is not arithmetic, and recall that $\mathrm{E}\left(\hat{\xi}_{1}\right)=m>0$, which means that $\hat{\xi}$ satisfies the conditions of Theorems 1 and 2 of [5]. Hence, the family of processes $\left(X_{\theta(t)}, t \geq 0\right)$ under $\widehat{\mathrm{P}}_{y}^{\uparrow}$ for $y>0$ converges weakly with respect to the Skorokhod topology as $y \downarrow 0$ towards a PSSMP starting from 0 , which is $\left(X_{\theta(t)}, t \geq 0\right)$ under $\widehat{\mathrm{P}}^{\uparrow}$. Moreover, according to the aforementioned results, the latter process has the same semigroup as $\left(X_{\theta}, \widehat{\mathrm{P}}_{y}^{\uparrow}\right)$ for $y>0$.

From Theorem 1 of [2], the entrance law of $\left(X_{\theta}, \widehat{\mathrm{P}}^{\uparrow}\right)$ is given by

$$
\widehat{\mathbb{E}}^{\uparrow}\left(f\left(X_{\theta(t)}\right)\right)=\frac{1}{(\alpha-1) m} \mathrm{E}\left(I^{-1} f\left(\left(t I^{-1}\right)^{1 /(\alpha-1)}\right)\right)
$$

for every $t>0$ and every $f$ positive and measurable. Therefore, from the distribution of $I$ given in Lemma 1 and some basic calculations, we obtain (10).

Proof of Theorem 2. The entrance law of the process $\left(Y, \mathrm{P}^{\uparrow}\right)$ can again be constructed from general considerations found in Theorem 1 of [5]. However, we give a more direct construction appealing to properties of the CSBP with immigration.

The PSSMP Lamperti representation follows from Proposition 2, where now the underlying Lévy process is $\xi^{*}$. Now, note that the Lévy process $\xi^{*}$ satisfies the conditions of Theorems 1 and 2 of [5], i.e. that $\xi$ is not arithmetic and $m^{*}=\mathrm{E}\left(\xi_{1}^{*}\right)>0$. Hence, the family of processes $\left\{\left(Y_{t}, t \geq 0\right), \mathrm{P}_{x}^{\uparrow}\right\}$ for $x>0$ converges weakly with respect to the Skorokhod topology as $x$ goes to 0 towards a PSSMP starting from 0 , which is $\left(Y, \mathrm{P}^{\uparrow}\right)$. It is well known (see, for instance, [16]) that its entrance law is of the form

$$
\mathbb{E}^{\uparrow}\left(\exp \left\{-\lambda Y_{t}\right\}\right)=\exp \left\{-\int_{0}^{t} \phi\left(u_{s}(\lambda)\right) \mathrm{d} s\right\} .
$$

Solving (5) explicitly we find that

$$
u_{t}(\lambda)=\left(c_{+}(\alpha-1) t+\lambda^{-(\alpha-1)}\right)^{-1 /(\alpha-1)} .
$$

We obtain (11) from straightforward calculations, recalling that $\phi(\lambda)=c_{+} \alpha \lambda^{\alpha-1}$; thus, completing the proof. 
Remark 3. Referring again to Proposition 3 of [5], which says in our particular case that

$$
\mathbb{E}^{\uparrow}\left(\exp \left\{-\lambda Y_{1}\right\}\right)=\frac{1}{m^{*}(\alpha-1)} \mathrm{E}\left(\exp \left\{-\lambda\left(I^{\prime}\right)^{-1}\right\}\left(I^{\prime}\right)^{-1}\right) \quad \text { for } \lambda \geq 0,
$$

where $I^{\prime}$ is the exponential functional of $(\alpha-1) \xi^{*}$ and is given by

$$
I^{\prime}:=\int_{0}^{\infty} \exp \left\{-(\alpha-1) \xi_{s}^{*}\right\} \mathrm{d} s
$$

it follows that we may characterise the law of $I^{\prime}$ via the relation

$$
\mathrm{E}\left(\exp \left\{-\lambda\left(I^{\prime}\right)^{-1}\right\}\left(I^{\prime}\right)^{-1}\right)=m^{*}(\alpha-1)\left(1+c_{+}(\alpha-1) \lambda^{\alpha-1}\right)^{-\alpha /(\alpha-1)} \quad \text { for } \lambda \geq 0 .
$$

\subsection{Proofs of Theorems 3, 4, 5, and 6}

Proof of Theorem 3. Fix $x>0$. From Theorem 1 we deduce that

$$
\mathrm{P}_{x}\left(Y_{\left(T_{0}-t\right)^{-}}<f^{1 /(\alpha-1)}(t) \text { i.o. as } t \rightarrow 0\right)=\widehat{\mathrm{P}}^{\uparrow}\left(X_{\theta(t)}<f^{1 /(\alpha-1)}(t) \text { i.o. as } t \rightarrow 0\right) .
$$

Since $\left(X_{\theta}, \widehat{\mathrm{P}}^{\uparrow}\right)$ is a PSSMP with index $\alpha-1$ starting from 0 , it is clear that the process $\left(X_{\theta}^{\alpha-1}, \widehat{\mathrm{P}}^{\uparrow}\right)$ is a PSSMP with index 1 . Then, from Theorem 3 of [8], the above probability is equal to 0 or 1 according respectively to whether

$$
\int_{0^{+}} \operatorname{Pr}\left(I>\frac{t}{f(t)}\right) \frac{\mathrm{d} t}{t}
$$

is finite or infinite. In order to get our result, it is enough to show that

$$
\operatorname{Pr}(I>t) \sim\left(c_{+}(\alpha-1) t\right)^{-1 /(\alpha-1)} \quad \text { as } t \rightarrow+\infty .
$$

From Lemma 1 and with the change of variable $h=\left(c_{+}(\alpha-1) t\right)^{-1 /(\alpha-1)}$, we have

$$
\lim _{t \rightarrow+\infty} \frac{\operatorname{Pr}(I>t)}{\left(c_{+}(\alpha-1) t\right)^{-1 /(\alpha-1)}}=\lim _{h \rightarrow 0} \frac{1-\mathrm{e}^{-h}}{h}=1,
$$

which establishes (18) and, hence, completes the proof.

Proof of Theorem 4. Here, we shall apply Theorem 1 of [22] to the process $\left(X_{\theta}^{\alpha-1}, \widehat{\mathrm{P}}^{\uparrow}\right)$. First, we note again that from Theorem 1 we have the following equality:

$$
\begin{aligned}
& \mathrm{P}_{x}\left(Y_{\left(T_{0}-t\right)^{-}}>(1+\varepsilon) f^{1 /(\alpha-1)}(t) \text { i.o. as } t \rightarrow 0\right) \\
& \quad=\widehat{\mathrm{P}}^{\uparrow}\left(X_{\theta(t)}>(1+\varepsilon) f^{1 /(\alpha-1)}(t) \text { i.o. as } t \rightarrow 0\right) .
\end{aligned}
$$

Hence, according to Theorem 1(i) of [22] and noting that the process $\left(X_{\theta}, \widehat{\mathrm{P}}^{\uparrow}\right)$ has no positive jumps, the right-hand side of the above equality is equal to 0 for all $\varepsilon>0$ if

$$
\int_{0+} \exp \left\{-\left(\frac{c_{+}(\alpha-1) t}{f(t)}\right)^{-1 /(\alpha-1)}\right\} \frac{\mathrm{d} t}{t}<\infty .
$$

In order to prove part (ii), we note that from Theorem 1 we have

$$
\begin{aligned}
& \mathrm{P}_{x}\left(Y_{\left(T_{0}-t\right)^{-}}>(1-\varepsilon) f^{1 /(\alpha-1)}(t) \text { i.o. as } t \rightarrow 0\right) \\
& \quad=\widehat{\mathrm{P}}^{\uparrow}\left(X_{\theta(t)}>(1-\varepsilon) f^{1 /(\alpha-1)}(t) \text { i.o. as } t \rightarrow 0\right) .
\end{aligned}
$$


Hence, applying Theorem 1(ii) of [22], we find that the above probability is equal to 1 for all $\varepsilon>0$ if

$$
\int_{0+} \exp \left\{-\left(\frac{c_{+}(\alpha-1) t}{f(t)}\right)^{-1 /(\alpha-1)}\right\} \frac{\mathrm{d} t}{t}=\infty .
$$

This completes the proof.

Proof of Theorem 5. From Lemma 1 we have

$$
-\log \operatorname{Pr}(I \leq t)=\left(c_{+}(\alpha-1) t\right)^{-1 /(\alpha-1)} .
$$

This fulfills condition (6.19) of Theorem 6 of [23] and, hence, directly applying the aforementioned result we deduce the LIL (12). Now, the time-reversed process $\left(Y_{\left(T_{0}-t\right)^{-}}, 0 \leq t \leq T_{0}\right)$ under $\mathrm{P}_{x}$ is a PSSMP starting from 0 with no positive jumps and its upper envelope is described by (12); then, from Theorem 8 of [23], the reflected process $\left((Y-\underline{Y})_{\left(T_{0}-t\right)^{-}}, 0 \leq t \leq T_{0}\right)$ also satisfies the same LIL (13).

The following result is crucial for the proof of Theorem 6 and can be seen as a corollary of Proposition 1. Recall the definition

$$
I^{\prime}:=\int_{0}^{\infty} \exp \left\{-(\alpha-1) \xi_{s}^{*}\right\} \mathrm{d} s
$$

Corollary 2. There is a positive constant $C$ which depends only on $\alpha$ such that

$$
\operatorname{Pr}\left(I^{\prime}>t\right) \sim C t^{-1 /(\alpha-1)} \text { as } t \rightarrow \infty .
$$

Proof. According to Lemma 4 of [25], if $\eta$ is a nonarithmetic Lévy process which drifts towards $-\infty$ and satisfies Cramér's condition for some $\theta>0$, i.e. $\operatorname{E}\left(\exp \left\{\theta \eta_{1}\right\}\right)=1$, then, for $\beta>0$,

$$
\operatorname{Pr}\left(\int_{0}^{\infty} \exp \left\{\beta \eta_{s}\right\} \mathrm{d} s>t\right) \sim K t^{-\theta / \beta} \quad \text { as } t \rightarrow \infty,
$$

where $K$ is a nonnegative constant which depends on $\beta$. Hence, recalling Proposition 1, we may apply Lemma 4 of [25] for the process $-\xi^{*}$ and obtain

$$
\operatorname{Pr}\left(I^{\prime}>t\right) \sim C t^{-1 /(\alpha-1)} \text { as } t \rightarrow \infty,
$$

where $C$ is a nonnegative constant which depends on $\alpha$.

Proof of Theorem 6. From Theorem 3 of [8] we have

$$
\mathrm{P}^{\uparrow}\left(Y_{t}<f^{1 /(\alpha-1)}(t) \text { i.o. as } t \rightarrow 0\right)=0 \text { or } 1
$$

according respectively to whether

$$
\int_{0+} \operatorname{Pr}\left(I^{\prime}>\frac{t}{f(t)}\right) \frac{\mathrm{d} t}{t}
$$

is finite or infinite. Hence, from Corollary 2, we obtain the desired result.

The integral test at $+\infty$ is proven similarly. 


\subsection{Proofs of Theorems 7 and 8}

Proof of Theorem 7. From Theorem 1 and since $X$ has no negative jumps, it is clear that

$$
\left.\left\{\left(Y_{\left(T_{0}-t\right)-}, T_{0}-U_{y} \leq t \leq T_{0}\right), \mathrm{P}_{x}\right)\right\} \stackrel{\mathrm{D}}{=}\left\{\left(X_{\theta(t)}, A_{\tau_{y}^{+}} \leq t<A_{\sigma_{x}}\right), \widehat{\mathrm{P}}^{\uparrow}\right\},
$$

where $\tau_{y}^{+}=\inf \left\{t>0: X_{t}>y\right\}$. On the other hand, by Theorem 1 of [7] we have, for $z \leq y$,

$$
\widehat{\mathrm{P}}_{y}^{\uparrow}\left(\inf _{t \geq 0} X_{t} \geq z\right)=\widehat{\mathrm{P}}_{y}^{\uparrow}\left(\inf _{0 \leq t \leq \sigma_{x}} X_{t} \geq z\right)=\frac{W(y-z)}{W(y)} .
$$

Hence, from (19), the above formula, and the Markov property of $\left(X, \widehat{\mathrm{P}}^{\uparrow}\right)$, the first statement of the theorem follows.

Next we remark that, for spectrally negative stable processes of index $\alpha \in(1,2]$, it is known that the scale function $W(x)$ is proportional to $x^{\alpha-1}$. Secondly, let $U_{y}=\sup \left\{t \geq 0: Y_{t} \geq y\right\}$, and note, again using the PSSMP Lamperti representation of $\left(Y, \mathrm{P}_{x}\right)$, that

$$
\operatorname{Pr}\left(\inf _{0 \leq t \leq D_{u}} \xi_{t} \geq v\right)=\mathrm{P}_{x}\left(\inf _{0 \leq t \leq U_{y}} Y_{t} \geq z\right)
$$

where $v=\log (z / x), u=\log (y / x)$, and $z \leq y$. Hence, from Corollary 1 we have

$$
\operatorname{Pr}\left(\inf _{0 \leq t \leq D_{u}} \xi_{t} \geq v\right)=\left(1-\mathrm{e}^{v-u}\right)^{\alpha-1},
$$

which establishes the conclusion.

Proof of Theorem 8 . We start by defining the family of PSSMPs $\widehat{X}^{(x)}$ whose PSSMP Lamperti representation is given by

$$
\widehat{X}^{(x)}=\left(x \exp \left\{\hat{\xi}_{\hat{\zeta}^{*}\left(t / x^{\alpha-1}\right)}^{*}\right\}, 0 \leq t \leq x^{\alpha-1} I^{\prime}\right), \quad x>0,
$$

where $\widehat{\xi}^{*}=-\xi^{*}$ and

$$
\hat{\zeta}^{*}(t)=\inf \left\{t: \int_{0}^{s} \exp \left\{(\alpha-1){\widehat{\xi^{*}}}_{u}\right\} \mathrm{d} u>t\right\} .
$$

Note that the random variable $x^{\alpha-1} I^{\prime}$ corresponds to the first time at which the process $\widehat{X}^{(x)}$ hits 0 ; moreover, for each $x>0$, the process $\widehat{X}^{(x)}$ hits 0 continuously.

We now set

$$
U_{y}^{-}=\sup \left\{t \geq 0: Y_{t} \leq y\right\} \quad \text { and } \quad \Gamma=Y_{U_{y}^{-}-} .
$$

According to Proposition 1 of [8], the law of the process $\widehat{X}^{(x)}$ killed when hitting 0 is a regular version of the law of the process $\left\{\left(Y_{\left(U_{y}^{-}-t\right)^{-}}, 0 \leq t \leq U_{y}^{-}\right), \mathrm{P}^{\uparrow}\right\}$ conditionally on $\{\Gamma=x\}, x \in[0, y]$. Hence, the latter process is equal in law to

$$
\left(\Gamma \exp \left\{\hat{\xi}_{\hat{\zeta}^{*}\left(t / \Gamma^{\alpha-1}\right)}^{*}\right\}, 0 \leq t \leq \Gamma^{\alpha-1} I^{\prime}\right)
$$

and $\hat{\xi}^{*}$ is independent of $\Gamma$. We deduce that

$$
\mathrm{P}^{\uparrow}\left(\sup _{0 \leq s<U_{y}^{-}} Y_{s} \leq z\right)=\operatorname{Pr}\left(\sup _{s \geq 0} \hat{\xi}_{s}^{*} \leq \log \left(\frac{z}{\Gamma}\right)\right) .
$$


On the one hand, it is a well-established fact that the all-time supremum of a spectrally negative Lévy process which drifts to $-\infty$ is exponentially distributed with parameter equal to the largest root of its Laplace exponent. In particular, for $x \geq 0$,

$$
\operatorname{Pr}\left(\sup _{s \geq 0} \hat{\xi}_{s}^{*} \leq x\right)=1-\mathrm{e}^{-x}
$$

Note that, by inspection of $\Psi^{*}(\theta)$, the largest root is clearly $\theta=1$ (there are at most two, and one of them is always $\theta=0$ ). On the other hand, from the above discussion, the random variables $\widehat{\xi^{*}}$ and $\Gamma$ are independent. Hence,

$$
\mathrm{P}^{\uparrow}\left(\sup _{0 \leq s \leq U_{y}^{-}} Y_{s} \leq z\right)=\mathrm{E}\left(1-\frac{\Gamma}{z}\right) .
$$

Therefore, in order to complete the proof, it is enough to show that $\mathrm{E}(\Gamma)=y / \mathrm{m}^{*}$.

We thus momentarily turn our attention to describing the law of $\Gamma$. Let $H=\left(H_{t}, t \geq 0\right)$ be the ascending ladder height process associated to $\xi^{*}$ (see Chapter VI of [1] for a formal definition), and denote by $v$ its Lévy measure. According to Lemma 1 of [8], the law of $\Gamma$ is characterised as follows:

$$
\log \left(y^{-1} \Gamma\right) \stackrel{\mathrm{D}}{=}-\mathcal{Z}
$$

where $\mathcal{U}$ and $\mathcal{Z}$ are independent random variables, $\mathcal{U}$ is uniformly distributed over [0, 1$]$, and the law of $\mathcal{Z}$ is given by

$$
\operatorname{Pr}(\mathcal{Z}>u)=\mathrm{E}\left(H_{1}\right)^{-1} \int_{(u, \infty)} s v(\mathrm{~d} s), \quad u \geq 0 .
$$

We may now compute

$$
\begin{aligned}
\mathrm{E}(\Gamma) & =y \int_{(0, \infty)} \int_{0}^{1} \mathrm{e}^{-u z} \mathrm{~d} u \operatorname{Pr}(\mathcal{Z} \in \mathrm{d} z) \\
& =y \int_{(0, \infty)} \frac{1}{z}\left(1-\mathrm{e}^{-z}\right) \operatorname{Pr}(\mathcal{Z} \in \mathrm{d} z) \\
& =\frac{y}{\mathrm{E}\left(H_{1}\right)} \int_{(0, \infty)}\left(1-\mathrm{e}^{-z}\right) v(\mathrm{~d} z)
\end{aligned}
$$

Next note that, since $\Psi^{*}(\theta)$ has its largest root at $\theta=1$, the Wiener-Hopf factorisation for the process $\hat{\xi}^{*}$ must necessarily take the form $\Psi^{*}(\theta)=(\theta-1) \phi(\theta)$ for $\theta \geq 0$, where $\phi(\theta)$ is the Laplace exponent of the descending ladder height process of $\hat{\xi}^{*}$. Note that $\phi$ has no killing term (i.e. $\phi(0)=0$ ) as $\hat{\xi}^{*}$ drifts to $-\infty$. Moreover, $\phi$ has no drift term as $\hat{\xi}^{*}$ has no Gaussian component (cf. [9, p. 175]). The latter two observations imply that

$$
\int_{(0, \infty)}\left(1-\mathrm{e}^{-z}\right) v(\mathrm{~d} z)=\phi(1)=\left.\frac{\Gamma(\theta-1+\alpha)}{(\theta-1) \Gamma(\theta-1) \Gamma(\alpha)}\right|_{\theta=1}=1 .
$$

Note also that $-m^{*}=\mathbb{E}\left(\hat{\xi}_{1}^{*}\right)=\Psi^{* \prime}(0+)=-\phi^{\prime}(0+)=-\mathbb{E}\left(H_{1}\right)$. Putting the pieces together in (20) completes the proof. 


\section{Concluding remarks on quasistationarity}

We conclude this paper with some brief remarks on a different kind of conditioning of CSBPs to (7) which results in a so-called quasistationary distribution for the special case of the self-similar CSBP. Specifically, we are interested in establishing the existence of normalisation constants $\left\{c_{t}: t \geq 0\right\}$ such that the weak limit

$$
\lim _{t \uparrow \infty} \mathrm{P}_{x}\left(\frac{Y_{t}}{c_{t}} \in \mathrm{d} z \mid T_{0}>t\right)
$$

exists for $x>0$ and $z \geq 0$.

Results of this kind have been established in [17] for CSBPs for which the underlying spectrally positive Lévy process has a second moment; see also [20]. In the more general setting, Pakes [21] formulated conditions for the existence of such a limit and characterised the resulting quasistationary distribution. The result below shows that in the self-similar case we consider in this paper, an explicit formulation of the normalisation sequence $\left\{c_{t}: t \geq 0\right\}$ and the limiting distribution is possible.

Lemma 2. Fix $\alpha \in(1,2]$. For all $x \geq 0$, with $c_{t}=\left(c_{+}(\alpha-1) t\right)^{1 /(\alpha-1)}$,

$$
\lim _{t \uparrow \infty} \mathbb{E}_{x}\left(\exp \left\{-\frac{\lambda Y_{t}}{c_{t}}\right\} \mid T_{0}>t\right)=1-\frac{1}{\left(1+\lambda^{-(\alpha-1)}\right)^{1 /(\alpha-1)}} .
$$

Proof. The proof pursues a similar line of reasoning to the aforementioned references [17], [20], [21]. From (4), it is straightforward to deduce that

$$
\lim _{t \uparrow \infty} \mathbb{E}_{x}\left(1-\exp \left\{-\frac{\lambda Y_{t}}{c_{t}}\right\} \mid T_{0}>t\right)=\lim _{t \uparrow \infty} \frac{u_{t}\left(\lambda / c_{t}\right)}{u_{t}(\infty)},
$$

if the limit on the right-hand side exists. However, since $\psi(\lambda)=\lambda^{\alpha}$, it is easily deduced from (5) that

$$
u_{t}(\lambda)=\left(c_{+}(\alpha-1) t+\lambda^{-(\alpha-1)}\right)^{-1 /(\alpha-1)},
$$

and the result follows after a straightforward calculation.

Although quasistationarity in the sense of 'conditioning to stay positive' does not make sense in the case of the CSBP with immigration, $\left(Y, \mathrm{P}_{x}^{\uparrow}\right)$, it appears that the normalising constants $\left\{c_{t}: t \geq 0\right\}$ serve a purpose to obtain the convergence in distribution below. A similar result is obtained of [17] for CSBPs with immigration whose underlying Lévy process has finite variance.

Lemma 3. Fix $\alpha \in(1,2]$. For all $x \geq 0$, with $c_{t}=\left(c_{+}(\alpha-1) t\right)^{1 /(\alpha-1)}$,

$$
\lim _{t \uparrow \infty} \mathbb{E}_{x}^{\uparrow}\left(\exp \left\{-\frac{\lambda Y_{t}}{c_{t}}\right\}\right)=\frac{1}{\left(\lambda^{(\alpha-1)}+1\right)^{\alpha /(\alpha-1)}} .
$$

Proof. We follow the ideas of [17], in which it was shown that $\left(Y, \mathrm{P}_{x}^{\uparrow}\right)$ may also be obtained as the Doob $h$-transform of the process $(Y, \mathrm{P})$ with $h(x)=x$. That is to say,

$$
\mathbb{E}_{x}^{\uparrow}\left(\exp \left\{-\lambda Y_{t}\right\}\right)=\mathbb{E}_{x}\left(\frac{Y_{t}}{x} \exp \left\{-\lambda Y_{t}\right\}\right)
$$


Differentiating (4) implies that

$$
\mathbb{E}_{x}^{\uparrow}\left(\exp \left\{-\lambda Y_{t}\right\}\right)=\exp \left\{-x u_{t}(\lambda)\right\} \frac{\psi\left(u_{t}(\lambda)\right)}{\psi(\lambda)} .
$$

Substituting in the necessary expressions for $\psi$ and $u_{t}(\lambda)$, as well as replacing $\lambda$ by $\lambda / c_{t}$ in the previous formula, the result follows directly.

\section{Acknowledgements}

Both authors would like to thank Victor Rivero for many useful discussions as well as an anonymous referee whose careful reading of an earlier version of this paper led to significant improvements. This research was funded by EPSRC grant number EP/D045460/1.

\section{References}

[1] Bertoin, J. (1996). Lévy Processes. Cambridge University Press.

[2] Bertoin, J. AND Yor, M. (2002). The entrance laws of self-similar Markov processes and exponential functionals of Lévy processes. Potential Anal. 17, 389-400.

[3] Bingham, N. (1976). Continuous branching processes and spectral positivity. Stoch. Process. Appl. 4, $217-242$.

[4] Caballero, M. E. and Chaumont, L. (2006). Conditioned stable Lévy processes and the Lamperti representation. J. Appl. Prob. 43, 967-983.

[5] Caballero, M. E. and Chaumont, L. (2006). Weak convergence of positive self-similar Markov processes and overshoots of Lévy processes. Ann. Prob. 34, 1012-1034.

[6] Caballero, M. E., Pardo, J. C. and Pérez, J. L. (2008). On the Lamperti stable processes. Preprint. Available at http://arxiv.org/abs/0802.0851.

[7] Chaumont, L. and Doney, R. (2005). On Lévy processes conditioned to stay positive. Electron. J. Prob. 10, 948-961.

[8] Chaumont, L. And Pardo, J. C. (2006). The lower envelope of positive self-similar Markov processes. Electron. J. Prob. 11, 1321-1341.

[9] Chaumont, L., Kyprianou, A. E. and Pardo, J. C. (2008). Some explicit identities associated with positive self-similar Markov processes. To appear in Stoch. Process. Appl.

[10] Donati-Martin, C. AND Yor, M. (2007). Further examples of explicit Krein representations of certain subordinators. Publ. Res. Inst. Math. Sci. 2, 315-328.

[11] Grey, D. R. (1974). Asymptotic behaviour of continuous time, continuous state-space branching processes. J. Appl. Prob. 11, 669-677.

[12] Grimvall, A. (1974). On the convergence of sequences of branching processes. Ann. Prob. 2, 1027-1045.

[13] Itô, K. And McKean, H. P. (1965). Diffusion Processes and Their Sample Paths. Springer, Berlin.

[14] JiRina, M. (1958). Stochastic branching processes with continuous state space. Czech. Math. J. 8, $292-313$.

[15] Lambert, A. (2001). Arbres, excursions, et processus de Lévy complètement asymétriques. Doctoral Thesis, l'Université Pierre et Marie Curie.

[16] Lambert, A. (2002). The genealogy of continuous-state branching processes with immigration. Prob. Theory Relat. Fields 122, 42-70.

[17] Lambert, A. (2007). Quasi-stationary distributions and the continuous-state branching process conditioned to be never extinct. Electron. J. Prob. 12, 420-446.

[18] Lamperti, J. (1967). Continuous state branching processes. Bull. Amer. Math. Soc. 73, 382-386.

[19] LAmperti, J. (1972). Semi-stable Markov processes. I. Z. Wahrscheinlichkeitsth. 22, 205-225.

[20] LI, Z.-H. (2000). Asymptotic behaviour of continuous time and continuous-state branching processes. J. Austral. Math. Soc. 68, 68-84.

[21] PAKes, A. (1999). Conditional limit theorems for continuous time and state branching processes. Res. Rep. 9/99, Department of Mathematics and Statistics, The University of Western Australia.

[22] Pardo, J. C. (2006). On the future infimum of positive self-similar Markov processes. Stochastics 78, $123-155$.

[23] Pardo, J. C. (2008). The upper envelope of positive self-similar Markov processes. To appear in J. Theoret. Prob.

[24] Patie, P. (2007). Law of the exponential functional of a new family of one-sided Lévy processes via self-similar continuous-state branching processes with immigration and the Wright hypergeometric functions. Preprint. Available at http://arxiv.org/abs/0712.1115. 
[25] Rivero, V. M. (2005). Recurrent extensions of self-similar Markov processes and Cramér's condition. Bernoulli 11, 471-509.

[26] Roelly, S. and Rouault, A. (1989). Processus de Dawnson-Watanabe conditionné par le futur lointain. C. $R$. Acad. Sci. Paris 309, 867-872.

[27] Sato, K. I. (1999). Lévy Processes and Infinitely Divisible Distributions. Cambridge University Press.

[28] Silverstein, M. L. (1968). A new approach to local time. J. Math. Mech. 17, 1023-1054. 